\title{
PERCEPÇÃO AMBIENTAL DE MORADORES E FREQUENTADORES DA RESERVA EXTRATIVISTA MARINHA DE ITAIPU, MUNICÍPIO DE NITERÓI, RJ
}

Marina Cyrino Leal Coutinho ${ }^{1}$

Michelle Marchezan Farias de Mesquita ${ }^{2}$

Ana Carolina da Silva Braga ${ }^{3}$

Renata Magalhães Jou ${ }^{4}$

\section{Resumo}

A Reserva Extrativista Marinha de Itaipu (RESEX de Itaipu) é uma categoria de Unidade de Conservação de Uso Sustentável prevista no Sistema Nacional de Unidades de Conservação. A RESEX de Itaipu localiza-se na região oceânica do município de Niterói e visa proteger a pesca artesanal tradicional e os recursos naturais que se encontram ameaçados nesta localidade, e garantir a exploração sustentável dos mesmos. O presente estudo foi realizado na extensão da Praia de Itaipu, utilizando-se questionário estruturado e semiestruturado, além de observação direta e conversas informais com alguns moradores no entorno da RESEX e frequentadores do local. Foram entrevistadas 58 pessoas, sendo 21 moradores e 37 visitantes. Os resultados demonstraram que os moradores têm melhor conhecimento e envolvimento em relação à RESEX, diferentemente dos visitantes, embora não houvesse clareza em reconhecer a área de estudo como uma RESEX já implementada por lei. De um modo geral, pode-se afirmar que existe ainda uma falta de conhecimento dos moradores, bem como dos visitantes sobre a criação e funcionamento da RESEX de Itaipu. Apesar destes resultados, a maioria dos entrevistados declarou ter interesse em obter mais informações a respeito da RESEX de Itaipu, o que reflete a necessidade de se realizar divulgação sobre a implementação da unidade de conservação na região e de projetos de educação ambiental para o maior esclarecimento da população. Assim, o presente estudo teve o intuito de averiguar a percepção ambiental dos frequentadores da praia de Itaipu sobre a implementação da RESEX, de forma a contribuir como um dado informativo na elaboração do plano de gestão desta área.

Palavras chave: RESEX, uso sustentável, zona costeira.

\begin{abstract}
The extractive reserve (RESEX) is a conservation units category envisaged in the National Protected Areas System (SNUC), framed in the classification of the sustainable use group. The RESEX Itaipu is located in the oceanic region of Niterói and aims to protect traditional artisanal fisheries and natural resources that are threatened at this location, and ensure the sustainable exploitation of the same. This study was conducted on the extent of the Itaipu Beach, using structured and semi-structured questionnaire, and direct observation and informal conversations with some locals surrounding the RESEX and visitors of the place. 58 people, 21 residents and 37 visitors were interviewed. The results showed that in relation to the perception of residents, we observed greater involvement and knowledge of these at the

\footnotetext{
${ }^{1}$ Universidade Federal Fluminense - Bióloga - E-mail: marinacoutinho88@ gmail.com

${ }^{2}$ Universidade Federal Fluminense - Bióloga - E-mail: michelle.marchezan@gmail.com

${ }^{3}$ Universidade Federal Fluminense - Bióloga - E-mail: annacarolbr@gmail.com

${ }^{4}$ Universidade Federal Fluminense - Engenheira Agrícola - E-mail: renatajou@gmail.com
} 
expense of visitors, although there were no clarity in recognizing the study area as a RESEX implemented by law. In general, it can be said that there is still a lack of knowledge of the residents and visitors on the establishment and operation of RESEX Itaipu. Despite these results, the majority of respondents claimed to have interest in obtaining more information about the RESEX Itaipu, which reflects the need to carry out dissemination on the implementation of conservation unit in the region and environmental education projects for the further clarification of the population. The present study aimed to investigate the environmental perception of the regulars Itaipu beach on the implementation of RESEX in order to contribute as information given in the preparation of the management plan of this area.

Keywords: RESEX, sustainable use, coastal zone

\section{INTRODUÇÃO}

O bairro de Itaipu localiza-se na Região Oceânica do município de Niterói, com cerca de 500.000 habitantes e área de $133.916 \mathrm{~km}^{2}$ (IBGE, 2015). O bairro está inserido dentro do bioma de Mata Atlântica, considerado um hotspot - uma região de elevada biodiversidade, que se encontra sob ameaça extrema- sendo, portanto, de grande importância do ponto de vista ambiental (BRANDON et al., 2005). Além disso, possui grande diversidade de ambientes naturais, tais como laguna, ilhas, costões rochosos e praia (BRASIL, 2013).

A enseada de Itaipu é classificada como um ecossistema de praia arenosa, considerado um sistema altamente dinâmico e com grande biodiversidade. Este ecossistema também é importante na retenção e processamento de matéria orgânica e poluentes, e é considerada área de berçário e desova de muitas espécies, como peixes, crustáceos e tartarugas (VELOSO e NEVES, 2009).

As regiões de praia, devido sua importância socioambiental, são incluídas como zona costeira, considerada patrimônio nacional e, portanto, fazem parte do Programa Nacional de Gerenciamento Costeiro (PNGC), o qual é parte integrante da Política Nacional do Meio Ambiente e da Política Nacional para os Recursos do Mar. O PNGC visa minimizar a deterioração ao longo do litoral brasileiro, ordenando o uso dos recursos naturais e a ocupação da zona costeira, integrando as esferas de governo (Municipal, Estadual e Federal) e os atores sociais que atuam nesta zona. Contudo, a efetividade do mesmo ainda não é a ideal, pois a maioria das zonas costeiras não possuem um plano de gestão bem implementado (BRASIL, 1988; MONTEIRO-NETO e NETO, 2009).

Na praia de Itaipu existe uma comunidade de pescadores artesanais tradicionais que tem na arte da pesca sua fonte de reprodução cultural e social. A propósito, a pesca artesanal de Itaipu foi reconhecida, pela Lei $n^{\circ}$ 2.874/2012, como Patrimônio Cultural Imaterial da cidade de Niterói. Entretanto, devido à expansão urbana e imobiliária da região, somadas a pesca industrial e a poluição das águas marinhas, essa comunidade está sendo ameaçada e já apresentou uma redução significativa no número de pescadores (SEA, 2013).

A Reserva Extrativista (RESEX) é uma categoria de Unidade de Conservação de Uso Sustentável prevista no Sistema Nacional de Unidades de Conservação (SNUC), que tem 
como objetivo básico compatibilizar a conservação da natureza com o uso sustentável de parcela de seus recursos naturais, permitindo diferentes formas e níveis de utilização humana (BRASIL, 2000). A categoria RESEX é definida como:

“... uma área utilizada por populações extrativistas tradicionais, cuja subsistência baseia-se no extrativismo e, complementarmente, na agricultura de subsistência e na criação de animais de pequeno porte, e tem como objetivos básicos proteger os meios de vida e a cultura dessas populações, e assegurar o uso sustentável dos recursos naturais da unidade" (Art. 18, Lei no 9.985, Brasil, 2000).

Desse modo, a criação da RESEX Marinha de Itaipu visa proteger a pesca artesanal tradicional e os recursos naturais que se encontram ameaçados nesta localidade, e garantir a exploração sustentável dos mesmos. Juntamente ao Parque Estadual da Serra da Tiririca (PESET), formam uma grande área de proteção ambiental aos diferentes ecossistemas marinhos e terrestres (BRASIL, 2013).

O processo de criação da RESEX de Itaipu durou quase 20 anos. A criação da RESEX foi proposta primeiramente na década de 90, pelo o extinto Centro Nacional para o Desenvolvimento Sustentado de Populações Tradicionais (CNPT/IBAMA), que não chegou a termo. Este processo ficou suspenso por anos devido a vários entraves e conflitos de interesses entre os principais participantes (pescadores, entidades comunitárias e CNPT/IBAMA). Em 2010, a proposta foi encaminhada ao INEA, por entender que se enquadrava melhor à esfera estadual e somente em 2012, um grupo de pescadores artesanais de Itaipu reapresentou a proposta à SEA que, em união com o INEA e a UFF, decidiu retomar o processo de criação da RESEX. A partir dai, foram realizadas 15 reuniões públicas e 2 oficinas com objetivo de informação, planejamento, construção dos limites da RESEX e articulação com o grupo de pescadores, suas organizações representativas, instituições parceiras, entidades governamentais e a sociedade civil. Por fim, foi realizada uma Consulta Pública para a consolidação deste processo e no dia 30 de setembro de 2013, através do decreto $n^{\circ}$ 44.417, a RESEX de Itaipu entrou em vigor (BRASIL, 2013; SEA, 2013).

A UC em questão localiza-se na Região Oceânica do município de Niterói, com área total de aproximadamente 3.943,28 hectares (exclusivamente marinha). Sua maior parte compreende a enseada de Itaipu, que está abrigada do oceano pelo alinhamento das ilhas do Pai, da Mãe e da Menina, e é dividida em sua porção mediana pelo canal da lagoa de Itaipu. Também abrange a área marinha adjacente às praias de Camboinhas, Itacoatiara (que faz fronteira com o município de Maricá, onde se encontra diretamente com a porção marinha do PESET), Piratininga e a entrada da Baía de Guanabara. O limite ao sul da RESEX baseia-se principalmente na impossibilidade de avanço da pesca artesanal em mar aberto (Figura 1) (BRASIL, 2013; SEA, 2013). 


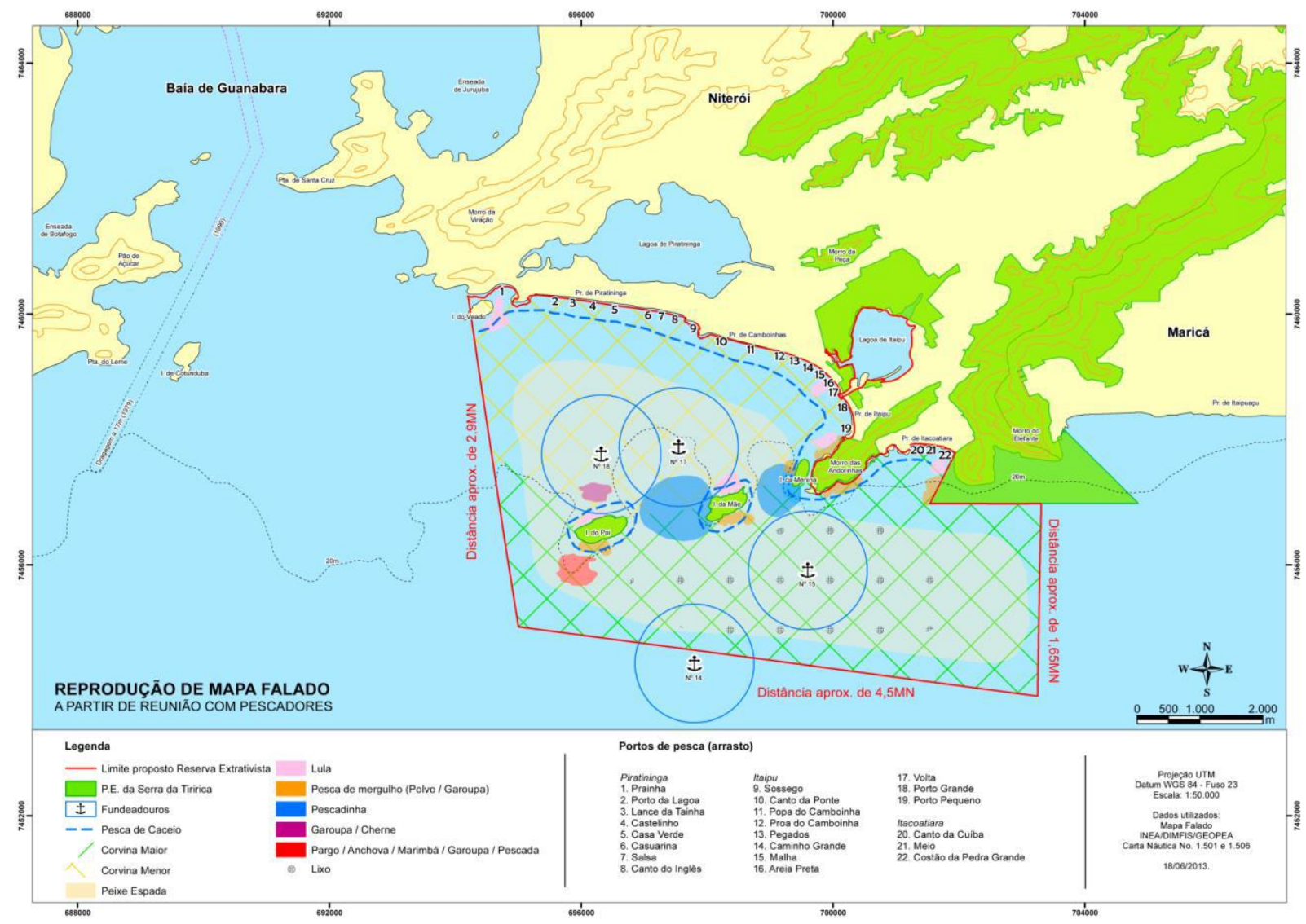

Figura 1: Mapa da área da RESEX de Itaipu delimitada em vermelho (SEA, 2013).

De acordo com o decreto $n^{\circ} 44.417$, a RESEX de Itaipu será regida por um Conselho Deliberativo, presidido pelo INEA, de forma participativa com representantes das associações de moradores e colônias de pesca de Niterói e adjacências, organizações governamentais e não-governamentais pertinentes, a Secretaria de Estado de Desenvolvimento Regional, Abastecimento e Pesca, e a UFF (BRASIL, 2013).

Para que exista uma gestão ambiental eficiente é necessário um estudo sobre a percepção ambiental dos envolvidos, direta e indiretamente, com a RESEX, uma vez que esta precisa ser monitorada e gerenciada de forma eficaz para manutenção da biodiversidade e para que os objetivos de sua criação sejam alcançados (GONÇALVES e HOEFFEL, 2012).

O presente estudo tem o objetivo avaliar a percepção ambiental dos frequentadores da praia de Itaipu, bem como determinar o nível de conhecimento dos mesmos sobre UCs e a recente implementação da RESEX de Itaipu, afim de que estas informações possam contribuir como um dado importante e estratégico na gestão da RESEX de Itaipu.

\section{MATERIAL E MÉTODOS}

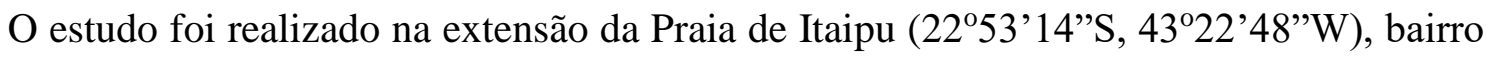
localizado na Região Oceânica da cidade de Niterói, no estado do Rio de Janeiro, utilizandose questionário estruturado e semiestruturado, além de observação direta e conversas 
informais com alguns moradores no entorno da Reserva Extrativista Marinha de Itaipu e frequentadores do local (Figura 2).

A linha de costa da praia de Itaipu ocupa uma extensão de $4,5 \mathrm{~km}$ e apresenta uma formação semicircular no sentido E-W. O perfil da praia é, na sua maior parte, íngreme e reflexivo, com profundidades entre 3 a $28 \mathrm{~m}$ e uma cobertura sedimentar homogênea, constituída predominantemente por areia média com alguns pontos de depósitos arenosos mais finos provenientes do canal de Itaipu (SALVADOR e SILVA, 2002).



Figura 2: Localização do ponto de amostragem na RESEX de Itaipu (em vermelho).

Os dados quantitativos foram organizados para análise usando o aplicativo Microsoft Excel 2007. As análises foram realizadas por meio de métodos da estatística descritiva, com auxílio de representações gráficas. Foram entrevistados moradores e visitantes com diferentes faixas etárias e níveis de escolaridade. A interpretação dos dados gerou gráficos que possibilitaram a análise preliminar sobre a percepção da população a respeito da RESEX de Itaipu, sua criação, bem como o envolvimento dos moradores na criação da UC e os possíveis benefícios.

\section{RESULTADOS E DISCUSSÃO}

Foram entrevistadas 58 pessoas, sendo 21 moradores e 37 visitantes, representando $36 \%$ e 64\%, respectivamente. A maioria dos moradores entrevistados são pescadores e/ou exercem atividades relacionadas, tais como, atravessador, limpador e vendedor de pescados. A faixa etária dos entrevistados variou de 15 a 65 anos, sendo a maioria da faixa de 40 e 59 anos (62 $\%)$, do sexo masculino (53\%) e residente do município de Niterói (62\%). 
Devido ao número relativamente baixo de entrevistados é possível que os dados não reflitam o perfil geral dos frequentadores da RESEX de Itaipu. No entanto, ainda assim o trabalho é válido, pois fornece uma visão preliminar para futuros estudos de investigação sobre como essas pessoas se relacionam com a RESEX de Itaipu, além de testar a metodologia empregada. Na literatura existem trabalhos semelhantes, com número baixo de questionários, que, entretanto, obtiveram resultados relevantes (SILVA et al., 2014; VÉRAS et al., 2014; SILVA e SILVA, 2013).

Quanto ao nível de escolaridade, as informações colhidas foram distribuídas nos seguintes graus de instrução: nível fundamental, nível médio e nível superior. Os entrevistados que possuíam níveis incompletos foram agrupados com os de mesmo nível completo. Assim, 23\% dos entrevistados possuíam ensino fundamental, 55\% ensino médio e $22 \%$ ensino superior. Não houve nenhum entrevistado sem escolaridade.

Quanto ao conhecimento do conceito de uma Unidade de Conservação (UC), $41 \%$ das pessoas responderam que sabiam o que era uma UC, enquanto $59 \%$ responderam que não sabiam. Dentre os entrevistados com resposta positiva, alguns indicavam que a UC que conheciam era o Parque Estadual Serra da Tiririca, enquanto outros desconheciam exemplos de UC apesar de saberem a definição de uma Unidade de Conservação. Esse resultado indica a distância que existe entre o assunto e a realidade da população e corrobora com os resultados obtidos por Ballarini et al. (2014) e Silva e Silva (2013).

Quando indagados se sabiam o que é uma RESEX, 53\% das pessoas responderam sim, enquanto $47 \%$ responderam não, mostrando resultados com valores próximos. Em contrapartida, quando perguntado aos entrevistados se conheciam alguma RESEX próxima, $22 \%$ responderam sim, enquanto a grande maioria (78\%) respondeu não (Figura 3A). Dentre os entrevistados que respondeu positivamente, a maioria eram moradores e/ou pescadores locais, que indicaram a RESEX de Itaipu e de Arraial do Cabo como exemplos, porém, não consideravam Itaipu como uma RESEX efetiva (Figura 3B). Enquanto os entrevistados que responderam negativamente, a maioria era visitante.

Os resultados aqui encontrados corroboram com os trabalhos de Carvalhido (2012) e Ballarini et al. (2014). Carvalhido (2012) menciona também a dificuldade na criação da RESEX de Itaipu, bem como a falta de envolvimento dos moradores do entorno. Entretanto, estes resultados não vão de encontro com a descrição do Estudo Técnico para a criação da RESEX Marinha de Itaipu (SEA, 2013), que destaca a grande participação do grupo de pescadores e entidades das comunidades do entorno no processo de criação da RESEX. De um modo geral, pode-se afirmar que existe ainda uma falta de conhecimento dos moradores, bem como dos visitantes sobre a criação e funcionamento da RESEX de Itaipu. 


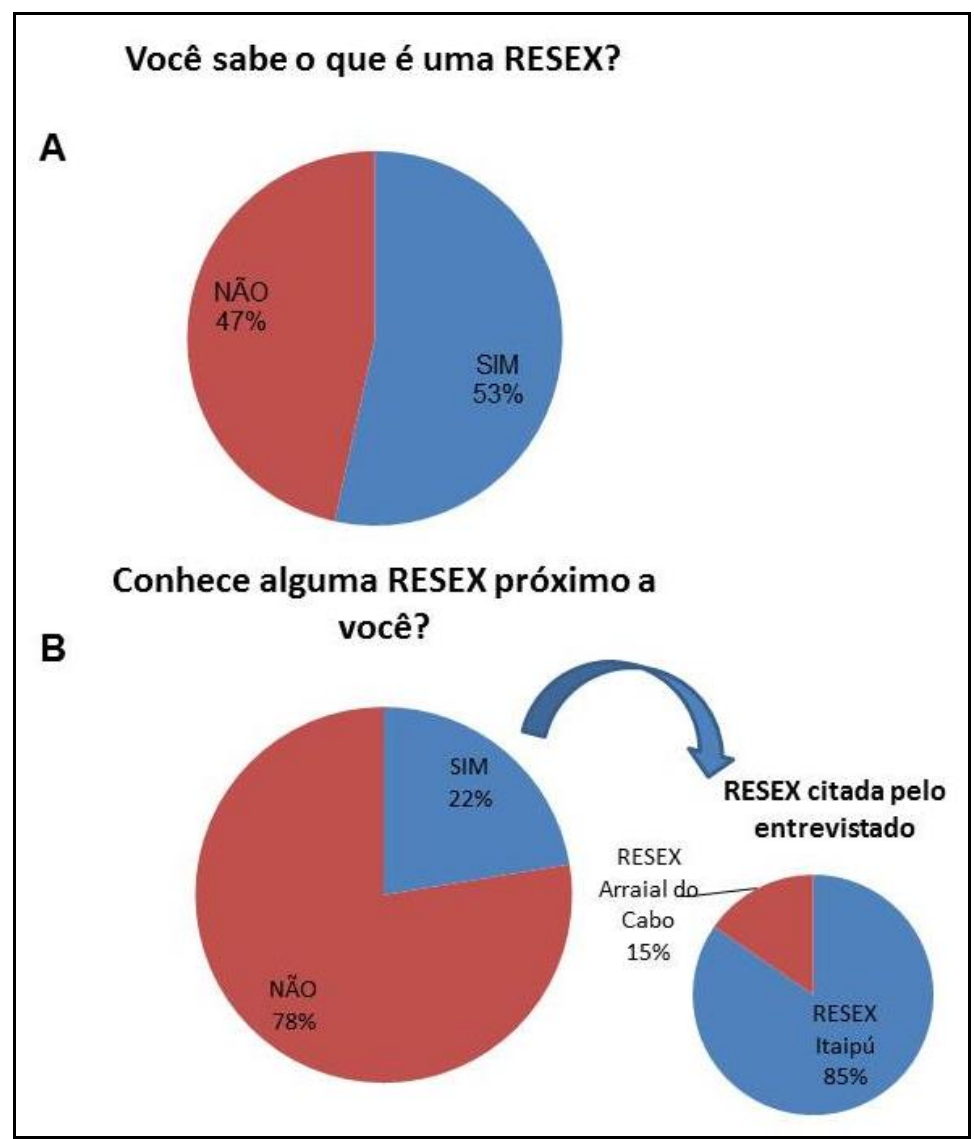

Figura 3: (A) Resposta dos entrevistados sobre o que é uma RESEX; (B) Resposta dos entrevistados sobre o conhecimento de uma RESEX próxima, indicando a RESEX que conheciam.

Quando abordados quanto às possíveis restrições e vantagens do uso de uma RESEX, as respostas foram divididas, $43 \%$ responderam sim e 57\% não (Figura 4). As restrições comentadas estavam relacionadas à limitação da área de pesca que poderia prejudicar o próprio pescador artesanal local. Já as vantagens, estavam relacionadas à sustentabilidade dos recursos pesqueiros para o pescador artesanal e proibição da pesca industrial. Ballarini et al. (2014) encontraram dados semelhantes para o estudo na RESEX Marinha do Estuário Amazônico. 


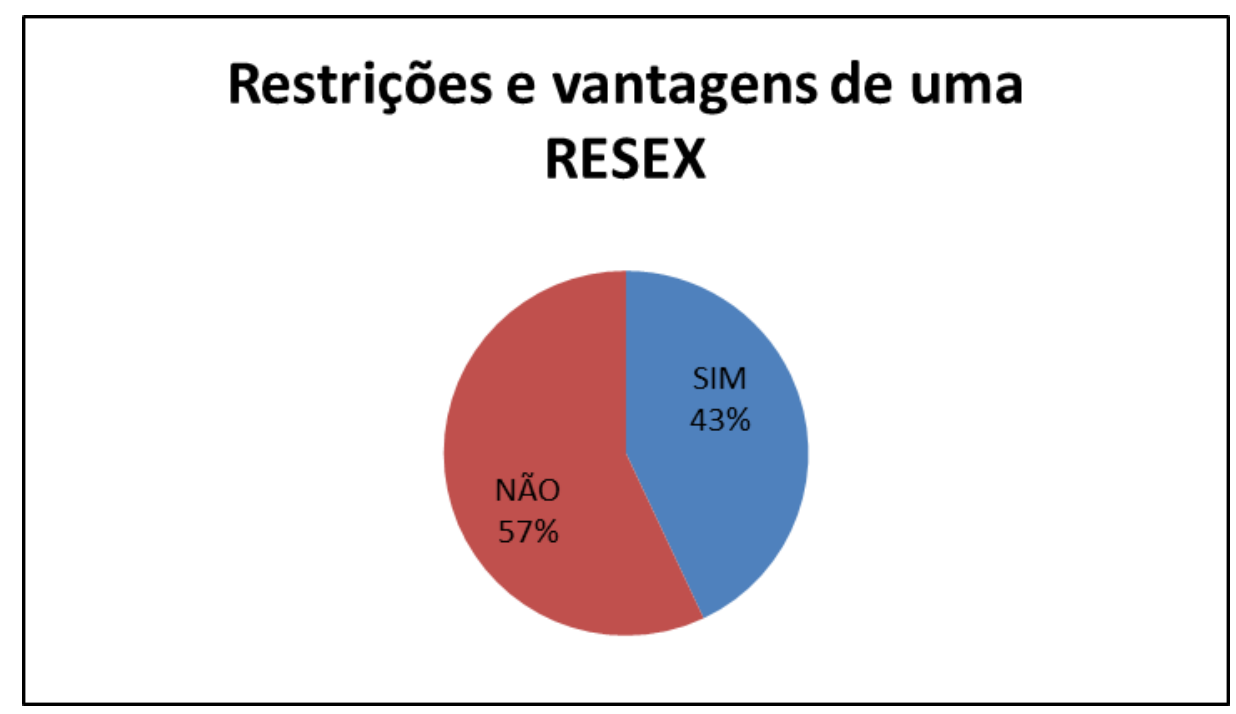

Figura 4: Resposta dos entrevistados sobre as restrições e vantagens de uma RESEX.

Para a criação de uma UC, cabe ao Poder Público realizar consulta pública a fim de fornecer informações adequadas à população local. A consulta pública tem como finalidade definir a localização, a dimensão e os limites geográficos mais adequados para a UC. A consulta pública da RESEX de Itaipu foi realizada no dia 30 de Julho de 2013 com a participação de diversos órgãos governamentais e não governamentais, além de associações e colônias de pescadores de Niterói e adjacências (SEA, 2013).

Ao serem indagados sobre a realização de alguma consulta pública para criação da RESEX de Itaipu, $83 \%$ dos entrevistados declararam não ter sido informado, enquanto $17 \%$ responderam ter tido acesso a esta informação. Apesar disso, SEA (2013) menciona que houve 15 reuniões públicas de mobilização, entre os anos de 2012 e 2013, com o objetivo de planejar e articular com o grupo de pescadores demandante, suas organizações representativas, entidades governamentais e da sociedade civil, apresentando e rediscutindo a proposta de criação da Reserva Extrativista Marinha de Itaipu.

Embora o resultado predominante tenha sido o não conhecimento da consulta pública da RESEX, vale ressaltar que a maioria dos entrevistados foi visitante, que utilizam a praia de Itaipu apenas para o lazer. Já os entrevistados que responderam sim, se enquadram no grupo dos pescadores locais e/ou moradores que participaram da criação da RESEX.

Apesar da falta de conhecimento encontrado por parte dos entrevistados, quando indagados sobre a possibilidade de se obter mais informações a respeito da RESEX de Itaipu, 97\% dos entrevistados declarou ter interesse, o que reflete a necessidade de se realizar divulgação sobre a implementação da RESEX na região e projetos de educação ambiental para o maior esclarecimento da população.

\section{CONCLUSÕES}

Os resultados demonstraram que quando avaliado a percepção dos visitantes sobre o conhecimento da RESEX de Itaipu, não há nenhum envolvimento com as questões 
relacionadas à sua criação, vantagens e restrições. Em relação à percepção dos moradores, foi possível observar um maior envolvimento e conhecimento, embora não houvesse clareza em reconhecer a área de estudo como uma RESEX já implantada por lei. Isso sugere que em termos práticos, há necessidade de uma implementação de um plano de manejo e/ou uma maior fiscalização, para que os objetivos de sua criação sejam alcançados.

Sabe-se que gerenciar o uso dos espaços inseridos em UCs é um desafio, pois não há um modelo específico que possa ser seguido e aplicado, uma vez que existem diversidades e especificidades locais e regionais. No entanto, a gestão participativa é essencial para a consolidação de uma UC e o envolvimento da sociedade é um elemento que deve ser considerado nas estratégias de conservação. Assim, o presente estudo teve o intuito de averiguar a percepção ambiental dos frequentadores da praia de Itaipu sobre a implementação da RESEX, de forma a contribuir como um dado informativo na elaboração do plano de gestão desta.

Dessa forma, ações de educação ambiental, oficinas e divulgação de informações são necessárias para a sensibilização de moradores e visitantes quanto à importância da região e a conservação dos recursos naturais marinhos da RESEX de Itaipu. Assegurando, desta forma, a preservação da área da RESEX e seu uso público responsável.

\section{REFERÊNCIAS BIBLIOGRÁFICAS}

BALLARINI D. H.; JIMENEZ E.; DOMINGUES D.; FERNANDES M. O olhar dos moradores de uma Resex marinha no estuário amazônico. In: Congresso Brasileiro de Oceanografia, 2014. Anais de resumos, Itajaí. Anais de resumos, Santa Catarina: Universidade do Vale do Itajaí - UNIVALI. 2014

BRANDON K.; FONSECA G.A.B; RYLANDS A.B.; SILVA J.M.C. Conservação brasileira: Desafios e oportunidades. Megadiversidade, Vol.1, $\mathrm{n}^{\circ} 1.2005$

BRASIL. Lei $\mathrm{n}^{\circ}$ 7.661, de 16 de maio de 1988. Institui o Programa Nacional de Gerenciamento Costeiro. 1988

BRASIL. Lei ${ }^{\circ}$ 9.985, de 18 de Julho de 2000. Institui o Sistema Nacional de Unidades de Conservação da Natureza. 2000

BRASIL. Decreto n44.417, 01 de outubro de 2013. Cria a Reserva Extrativista Marinha de Itaipu no Município de Niterói e dá outras providências. 2013

CARVALHIDO V. V. R. Do “direito à vez" à vez aos direitos: Conflitos e representações acerca do espaço e do trabalho no Canto de Itaipu. Dissertação de Mestrado. Programa de Pós-Graduação em Antropologia, Universidade Federal Fluminense. Niterói. 97p. 2012

GONÇALVES N.M.; HOEFFEL J.L.M. Percepção ambiental sobre unidades de conservação: Os conflitos em torno do parque estadual de Itapetinga - SP. Revista VITAS - Visões transdisciplinares sobre Ambiente e Sociedade. 2012 
IBGE - Instituto Brasileiro de Geografia e Estatística. Disponível em: < http://www.cidades.ibge.gov.br > Consultado em: 08/04/2015

MONTEIRO-NETO C.; NETO J. P. M. Biologia da Conservação Marinha. In: Pereira R.C. E Soares-Gomes A. (Eds.) Biologia Marinha, Editora Interciência, 2a edição, 631p. 2009

PRIMACK R.B; RODRIGUES E. Biologia da Conservação. Rio de Janeiro: Planta. 2001

SEA - Secretaria de Estado do Ambiente. Estudo técnico para a criação de Reserva Extrativista Marinha de Itaipu - RESEX Itaipu. 2013

SILVA M.; COSTA B.; COSTA P.M. Pesca, gestão dos recursos pesqueiros e desenvolvimento econômico: um estudo de caso na Resex Mãe Grande de Curuçá, Pará. In: Congresso Brasileiro de Oceanografia, 2014. Anais de resumos, Itajaí. Anais de resumos, Santa Catarina: Universidade do Vale do Itajaí - UNIVALI. 2014

SILVA V. M.; SILVA B. T. B. Percepção ambiental da comunidade do entorno do Parque Natural Municipal Barão de Mauá, Município de Magé, RJ. In: I Encontro Fluminense - Uso Público em Unidades de Conservação. Anais, Niterói: Universidade Federal Fluminense, n. 1, v, 1.2013

VELOSO V. G.; NEVES G. Praias arenosas. In: Pereira R.C. E Soares-Gomes A. (Eds.) Biologia Marinha, Editora Interciência, 2a edição, 631p. 2009

VÉRAS P. F.; TORRES H. S.; ALMEIDA Z. S.; SOUSA A. F. R.; CARDOSO A. S.; EVERTON L. S.; LICÁ I. C. L.; ALENCAR L. S.; FARIAS P. M. Aspectos sociais, conflitos de pesca e manejo na ilha dos lençóis, Cururupu - Ma. In: Congresso Brasileiro de Oceanografia, 2014. Anais de resumos, Itajaí. Anais de resumos, Santa Catarina: Universidade do Vale do Itajaí - UNIVALI. 2014 